NISTIR 8243

\title{
A Test Method for Measuring Response Robot Performance Under Attenuated Radio Conditions
}

Kenneth E. Kimble

Richard Candell

Kamel S. Saidi

This publication is available free of charge from:

https://doi.org/10.6028/NIST.IR.8243

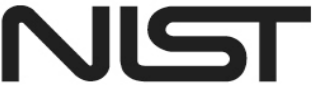

National Institute of Standards and Technology

U.S. Department of Commerce 
NISTIR 8243

\section{A Test Method for Measuring Response Robot Performance Under Attenuated Radio Conditions}

Kenneth E. Kimble

Richard Candell

Kamel S. Saidi

Intelligent Systems Division

Engineering Laboratory

This publication is available free of charge from:

https://doi.org/10.6028/NIST.IR.8243

May 2019

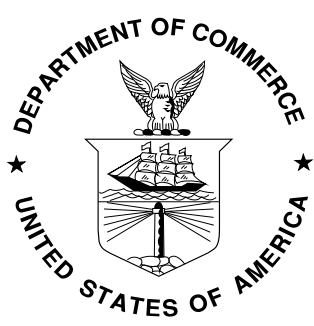

U.S. Department of Commerce Wilbur L. Ross, Jr., Secretary

National Institute of Standards and Technology Walter Copan, NIST Director and Undersecretary of Commerce for Standards and Technology 
Certain commercial entities, equipment, or materials may be identified in this document in order to describe an experimental procedure or concept adequately. Such identification is not intended to imply recommendation or endorsement by the National Institute of Standards and Technology, nor is it intended to imply that the entities, materials, or equipment are necessarily the best available for the purpose.

National Institute of Standards and Technology Interagency or Internal Report 8243

Natl. Inst. Stand. Technol. Interag. Intern. Rep. 8243, 16 pages (May 2019)

This publication is available free of charge from:

https://doi.org/10.6028/NIST.IR.8243 


\begin{abstract}
Emergency and disaster situations are unavoidable. Earthquakes, severe weather, bomb threats, and war are an unfortunate reality. Firefighters, bomb technicians, and urban search and rescue specialists are the first to respond in emergency situations. These first responders risk their lives where remotely operated robots would be more suited for the dangers. Emergency response robots are designed for remote operation implying a wireless connection between the controller and robot. The performance of the wireless communication connection between controller and robot is typically non-ideal. Reflective and absorptive properties of the operational environment and interference degrade the communication system, limiting range, reliability, and timeliness. An affordable and effective method for evaluating the performance of emergency response robots under specific attenuated radio conditions is proposed in this paper. The method may be useful for continuing work on a current standard: E2855-12 Standard Test Method for Evaluating Emergency Response Robot Capabilities: Radio Communication: Non-Line-of-Sight Range[3] currently being pursued by the American Society for Testing and Materials (ASTM) International Standards Committee on Homeland Security Applications
\end{abstract}

\title{
(E54.09).Key words
}

Decibel (dB); Operator Control Unit (OCU); Path loss; Attenuator; Radio attenuation; Radio communication; Test method; 


\section{Table of Contents}

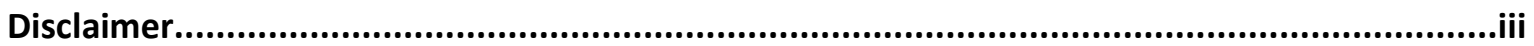

I. Introduction ............................................................................................. 1

II. Radio Attenuation....................................................................................... 1

III. Proposed Testing Procedure .......................................................................... 3

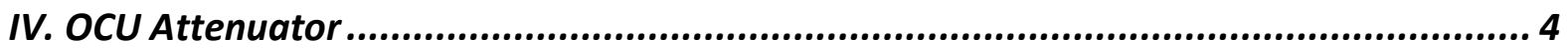

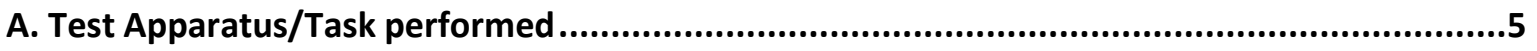

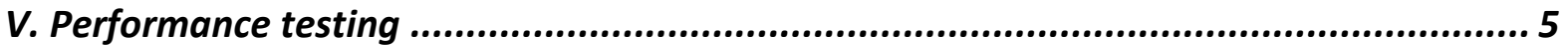

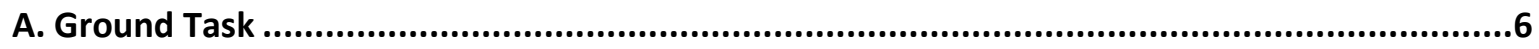

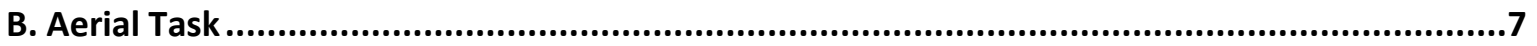

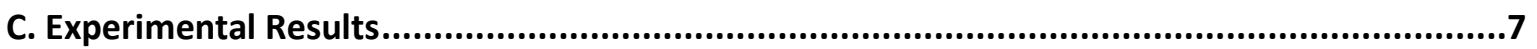

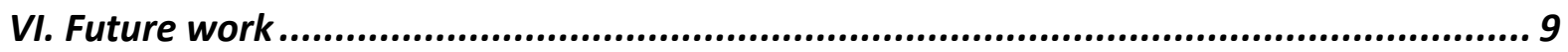

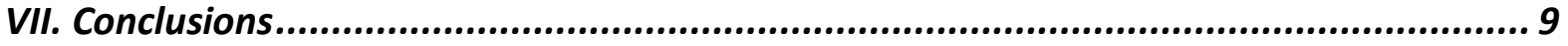

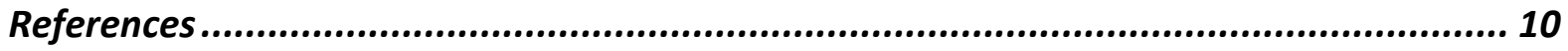

Table 1: Attenuation distance equivalence chart (Measures in meters, M) ............................. 2

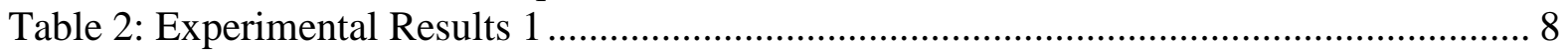

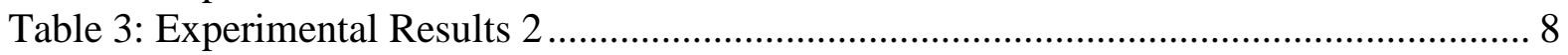

Figure 1: Example path loss curves for frequencies of $900 \mathrm{MHz}, 2400 \mathrm{MHz}$, and $5400 \mathrm{MHz} 2$

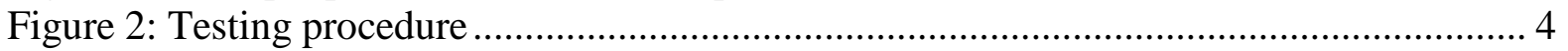

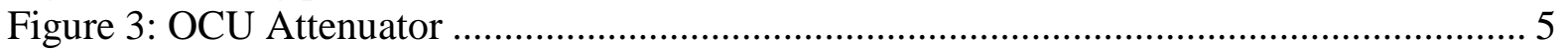

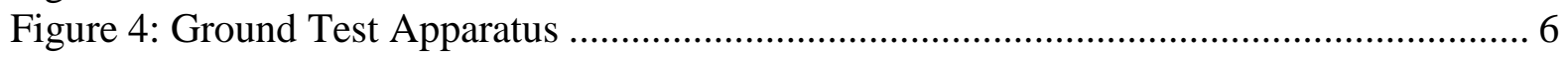

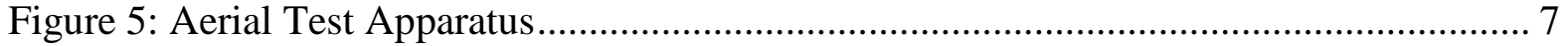

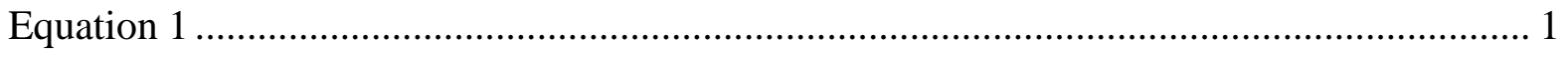

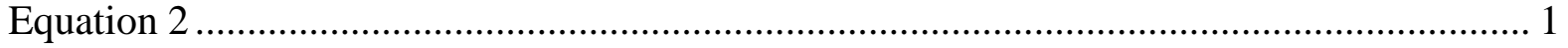




\section{Disclaimer}

Certain commercial equipment, instruments, or materials are identified in this paper in order to specify the experimental procedure adequately. Such identification is not intended to imply recommendation or endorsement by the National Institute of Standards and Technology, nor is it intended to imply that the materials or equipment identified are necessarily the best available for the purpose. 


\section{Introduction}

In a world where radio communications between operator and robot are so prevalent and so necessary, there needs to exist a way for an operator to understand the capabilities of their robot under mission specific environments. This understanding will allow them to better access their ability to operate given their specific mission parameters prior to beginning the mission. A test method is proposed in this paper that will quantitatively measure robot behavior under simulated non-ideal situations. The goal is to create a controlled and increasingly attenuated environment to measure the point at which a robot can no longer perform a task due to the loss in communications. Measuring the robot's loss in communications is difficult to quantify and so the measurements are based solely on the amount of attenuation in the test method and at what point the robot's performance is affected/disabled. The performance of the robot in a disaster scenario is not directly proportional to the results of this test method since certain factors such as specifications of the antennae or robot are not evaluated during the test. The radio attenuation equation is a representation of differing environmental obstacles, changes in distance, permeability of the air, and the amount of interference in the surroundings. The equation offers no information on the effects of such attenuation on a robot. This test method successfully measures and quantifies the point at which a robot is affected and/or fails a task due to the radio loss associated with attenuation. A secondary goal is to inform a user community of the importance of knowing when and how a robot will behave with communication issues, as it is vital information to have before entering an emergency situation. The measured results are a part of an ongoing exercise to create a standard test method that sufficiently measures attenuation as it affects a robot.

\section{Radio Attenuation}

Using Eq. 1 as specified in ITU-R P.1238.7 [1], radio wave propagation path loss may be calculated using

$$
\begin{gathered}
\text { Equation } 1 \\
\mathrm{~L}=20 \log 10(\mathrm{f})+10 \mathrm{n} \log 10(\mathrm{~d}) 28
\end{gathered}
$$

where $\mathrm{n}$, is the path loss exponent, $\mathrm{f}$ is frequency in $\mathrm{MHz}$, and $\mathrm{d}$ is distance in meters. This is a basic method of calculating path loss and will suffice for the purposes of robot performance evaluation. Communications systems are often evaluated using a log-normal path loss model which is calculated by

$$
\begin{gathered}
\text { Equation } 2 \\
\mathrm{~L}=\mathrm{L} 0+10 \mathrm{n} \log 10(\mathrm{~d} / \mathrm{d} 0)+\mathrm{Xg}
\end{gathered}
$$


where L0 is the path loss measured at a reference distance, $\mathrm{d} 0$, and $\mathrm{Xg}$ is a random variable with a distribution that models the type of fading environment. For a shadowing or slow fading environment, $\mathrm{Xg}$ will be a Gaussian with zero mean and standard deviation in $\mathrm{dB}$ resulting in a log-normal distribution. For multi-path environments, $\mathrm{Xg}$ is modeled as a Rician or Rayleigh distributed random variable [2].

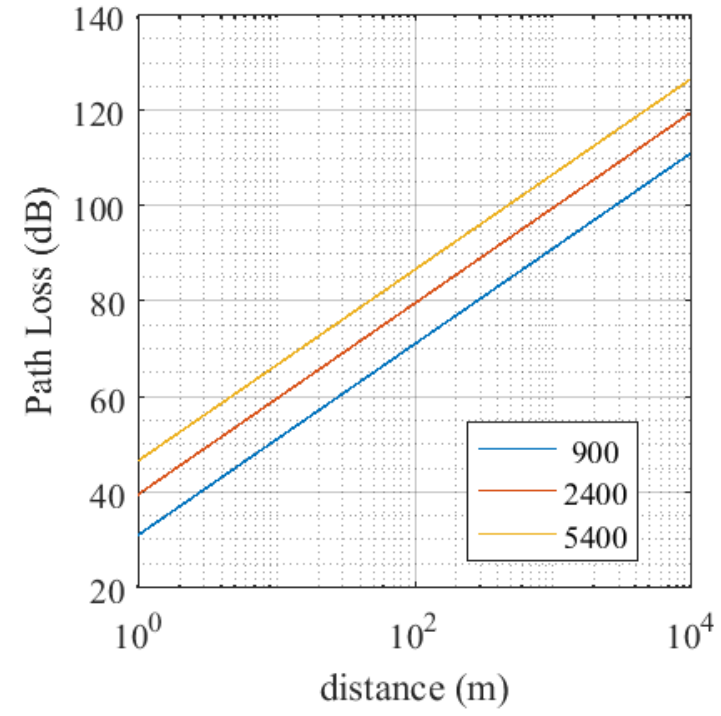

(a) $n=2.0$ Free-space

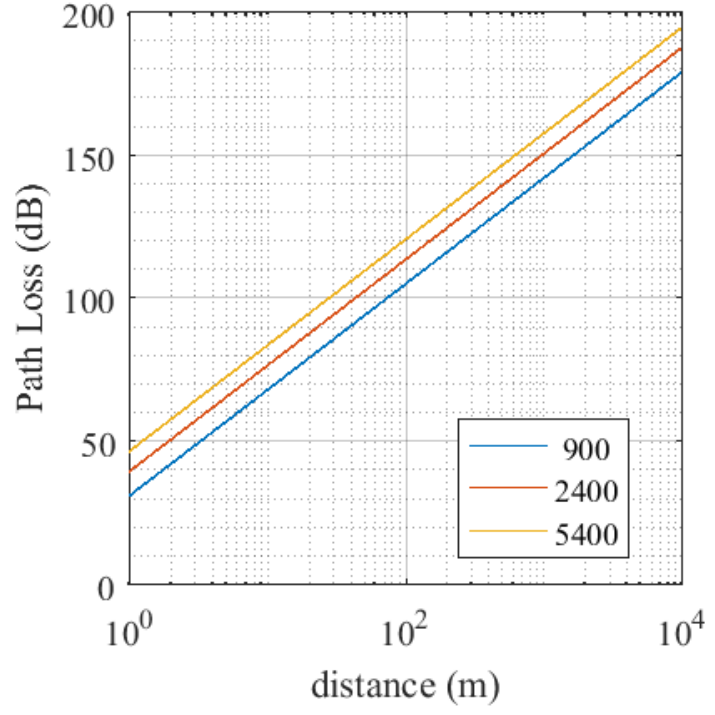

(b) $n=3.7$, Occluded industrial

Figure 1: Example path loss curves for frequencies of $900 \mathrm{MHz}, 2400 \mathrm{MHz}$, and $5400 \mathrm{MHz}$

Fig 1. Shows three different frequencies produced within two separate environments as an example of how the environment effects the path loss. The path loss for the Occluded industrial environment (b) starts higher from a zero distance and climbs faster as the distance increases when compared to the free space environment. Practically speaking, this translates to a much greater loss in communications over an equivalent distance in the free space environment.

Table I shows equivalent distance, in meters, with an external attenuator within common environments ranging from "Free Space" which includes little to no interferences up to "Industrial” which includes heavy interferences. Each environment is listed with a typical path loss exponent. A higher exponent indicates more rapid path loss; hence, the equivalent distance will be lower. The chart also exemplifies the impact of the path loss exponent to the radio channel. Slight changes in the exponent value will result in a large relative change in equivalent distance because of the exponential relationship between distance and path loss.

Table 1: Attenuation distance equivalence chart (Measures in meters, $\mathrm{M}$ )

\begin{tabular}{|c|c|c|c|c|c|c|}
\hline & \multicolumn{3}{|c|}{$2.4 \mathrm{GHz}$} & \multicolumn{3}{c|}{$5.4 \mathrm{GHz}$} \\
\hline Channel Gain, $\mathrm{dB}$ & Free Space & Office & Industrial & Free Space & Office & Industrial \\
\hline $\mathrm{dB}$ & $\mathrm{n}=2$ & $\mathrm{n}=3$ & $\mathrm{n}=3.7$ & $\mathrm{n}=2$ & $\mathrm{n}=3.1$ & $\mathrm{n}=2.9$ \\
\hline 0 & 0 & 0 & 0 & 0 & 0 & 0 \\
\hline
\end{tabular}




\begin{tabular}{|c|c|c|c|c|c|c|}
\hline 40 & 1.0 & 1.0 & 1.0 & 1.0 & 1.0 & 1.0 \\
\hline 80 & 104.7 & 22.2 & 12.4 & 104.7 & 20.1 & 24.7 \\
\hline 120 & 10466.2 & 478.5 & 148.9 & 10466.2 & 392.1 & 591.9 \\
\hline
\end{tabular}

\section{Proposed Testing Procedure}

A predetermined task is performed in an open environment and the results recorded as a baseline measurement. The Operator Control Unit (OCU) is the controller used by the operator to communicate controls with the robot. The OCU is attenuated using a box that completely encloses the controller separating it from the environment. The OCU Attenuator allows the test administrator to add layers of material known as Lossy Foam Absorber, made of high-density anechoic foam. The robot performance is tested at each layer of material. If the robot fails to complete the task, the experimental attenuation is recorded as the point of failure. If the robot completes the task but shows a decrease in performance, any effects are noted, and the testing continues. After the results of each task are recorded, a layer of attenuating material is added, measured, and recorded and the task is performed again. A goal of reaching $120 \mathrm{~dB}$ of loss and still operating is set as a benchmark to separate robot performance into pass/fail conditions for simplicity of testing. The procedure is shown in Fig. 2. 


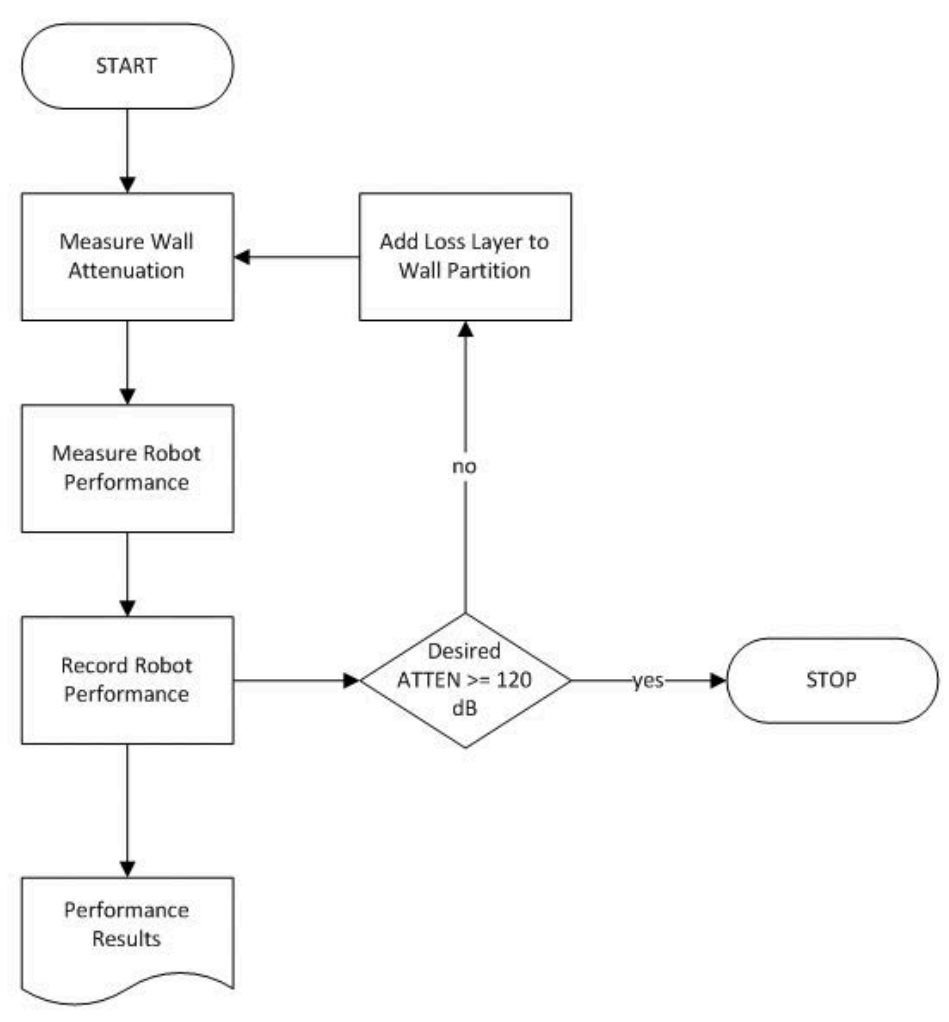

Figure 2: Testing procedure

\section{OCU Attenuator}

Fig. 3 shows the attenuation box: A $0.6 \mathrm{~m}$ box made of oriented strand board (OSB) and small pieces of 1.5 in x3.5 in lumber. Two 6-inch diameter holes are placed in the side of the box to allow the operator access to the controller. A High-Definition Multimedia Interface (HDMI) cable runs from the OCU inside the box, through one arm hole, to a nearby monitor outside the box so that the operator and test administrator can see the output display of the OCU. The attenuating material is placed around the box on all sides to increase the decibel of attenuation. The attenuating material was tested independently and shown to create approximately $40 \mathrm{~dB}$ of attenuation per sheet. Specific experimental measurements are shown in the test results for each layer. 


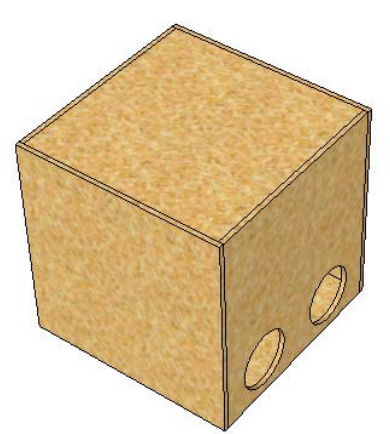

(a) Closed view

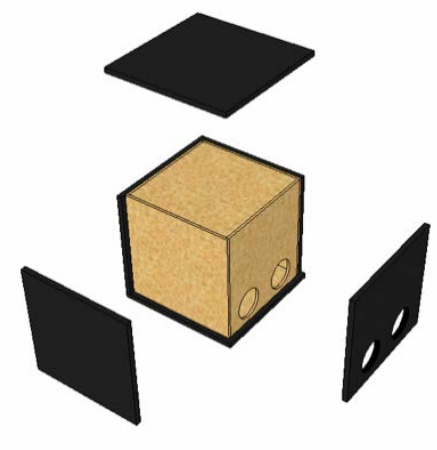

(b) Single layer

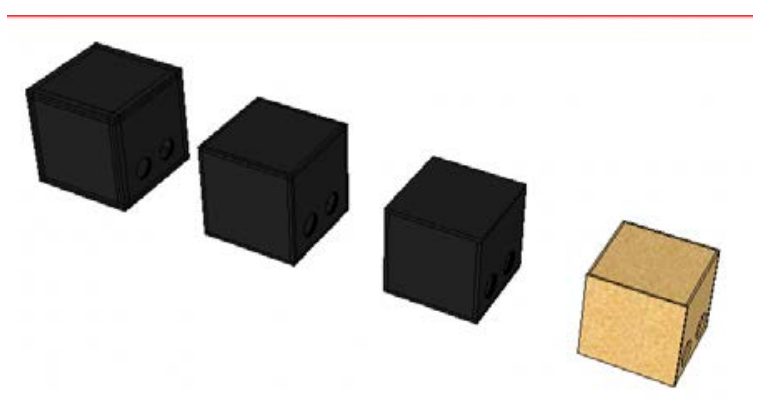

(c) Exploded, Three layers

Figure 3: OCU Attenuator

\section{A. Test Apparatus/Task performed}

The experiment was performed in an environment where multiple other tests were occurring at the same time. This had a direct effect on the performance of the robots used in this experiment. By measuring the performance of robots under the same environmental conditions, exterior effects can be factored out with the understanding that performance in a lower noise environment would improve. In order to compare relative capabilities of any robot under attenuation, repeatable test methods must be introduced. This will help evaluate the performance of the robots, quantify that performance, and indicate how the attenuation is directly affecting the robot's communications. Well known and regularly exercised aerial and ground test methods were used to evaluate the performance of the robots used during the experiment.

\section{Performance testing}

The test method procedures and apparatuses are currently being considered for standard test methods for response robots E54.09. It's important to recognize that the tests that were performed for this experiment are secondary measures and are not the only way to quantify 
robot performance under attenuation. The procedures of each test are shown below as well as the results of the testing performed and any outcomes from the experiment.

\section{A. Ground Task}

Fig. 4 shows the ground-based robot test apparatus and the environment that was used as the performance task for the ground robots. The operator was given 4 min to drive as many laps across the terrain as possible. The path across the terrain required the robot to traverse between the two blue posts in the form of an 'S' from end-zone to end-zone. First, the operator directs the robot in a forward motion to one end-zone, then, back to the starting endzone along the same path in reverse. The task was designed to expose the robot to complex terrain requiring higher levels of mobility. This also ensured a need for the operator to input multiple driving commands along the entire length of the course. The number of laps was recorded as the score for that run where a forward traverse was worth one point and a reverse traverse was worth one point. At the end of $4 \mathrm{~min}$, the operator must stop and only the total number of completed laps was counted.

The ground robot that was used for this experiment failed to respond to any commands from the OCU at the lowest level of attenuation due to surrounding initial conditions and as such was unable to perform the ground-based task that had been planned. No usable experimental results were obtained for the ground robots' section of this exercise.

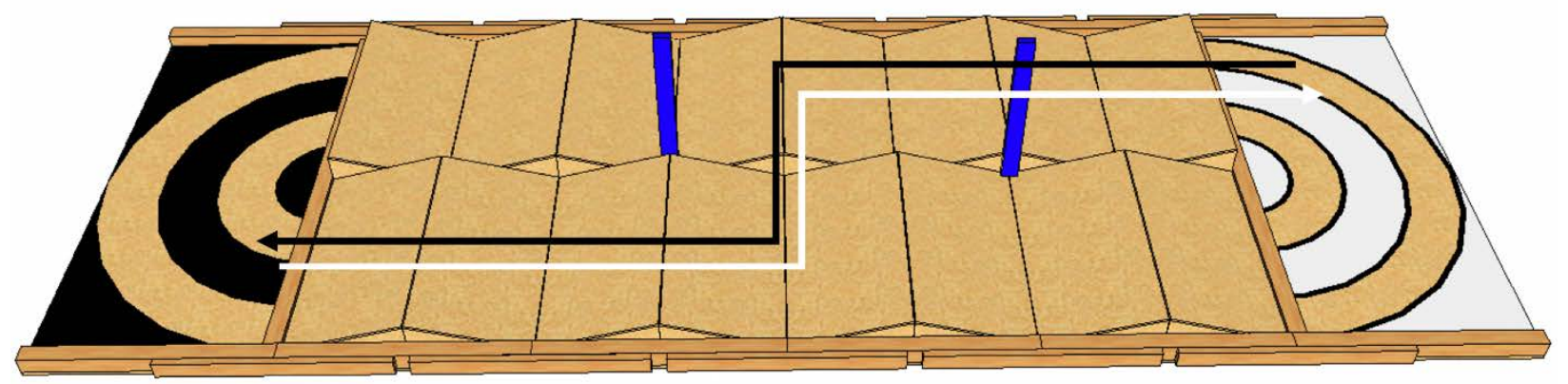

(a) Ground Test Environment

Figure 4: Ground Test Apparatus 


\section{B. Aerial Task}

Fig. 5 shows the aerial test apparatus and the environment that was used as the performance task for the aerial robots. The robot operator was asked to perform a task that exaggerates the aerial robot's capability to fly in compound flight patterns consisting of $x-y-z$ and yaw rotation. The test also required the ability to station keep (i.e. hover in place) while identifying a target at close range (less than $0.5 \mathrm{~m}$ ). This task was the most comprehensive for aerial mobility and as such it tested most of the aerial's performance capabilities.

When the robot operator was ready, a timer set for four minutes was started and the test began. The aerial robot took off from the launch/land zone and hovered at $1.2 \mathrm{~m}(4 \mathrm{ft})$ off the ground. When stable control was shown, the operator directed the aerial to the test apparatus. Beginning with the bottom-most target, the operator directed the aerial close enough to the Polyvinyl Chloride(PVC) pipe to reveal the target placed inside. The screen of the OCU must display the target completely such that all of the lines inside the pipe can be seen and counted in the same image; (Fig. 5). The operator was allowed to move on to the next target having gained one point. The operator moved to the next closest target from the bottom forcing the aerial robot to rotate around the apparatus. Then, the operator continued to each target moving up the apparatus until they had reached the top most target. When the operator had identified the top target of the test they were allowed to continue in reverse order descending back down the post for more points. When the 4-minute timer ended, the test was over, and the aerial was commanded to land back in the launch/landing zone.

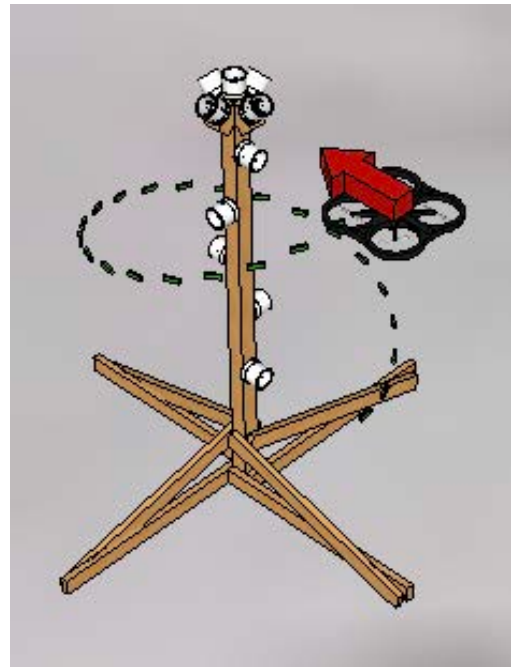

(a) Spiral test apparatus

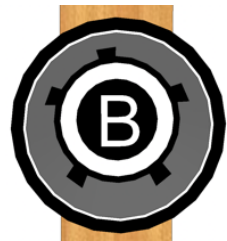

(b) Inspection target

Figure 5: Aerial Test Apparatus

\section{Experimental Results}

Data was recorded noting the theoretical value of attenuation and the measured value of attenuation using numbers $(-1,0,1,2,3)$, where -1 represents the procedure done without any 
OCU attenuator, 0 represents the OCU attenuator without any layers of material, and increasing whole numbers represent each layer of attenuation material added thereafter.

Table II shows the results of the first aerial test performed. The aerial had no issues under the measured $0 \mathrm{~dB}$ and $6 \mathrm{~dB}$ and scored 6 targets on both trials. The aerial showed control issues at a measured $36 \mathrm{~dB}$ of attenuation and crashed. After a second run the score improved to 7 targets showing that the operator had improved their proficiency as more trials were performed but had no control issues. This proved that any communications issues experienced were not hindering performance on the first run. The aerial failed to perform the test at a measured $72 \mathrm{~dB}$ and didn't respond to any commands at $110 \mathrm{~dB}$ of attenuation. This meant that between one layer of attenuating material and two layers the aerial lost the ability to fly reliably and at some point, after the measured $72 \mathrm{~dB}$ of attenuation (2 layers) but before $110 \mathrm{~dB}$ (3 layers), the aerial showed a complete loss of communications.

Table 2: Experimental Results 1

\begin{tabular}{|c|c|c|c|c|}
\hline Layers & Nom. dB & Measured dB & Performance & Notes \\
\hline-1 & 0 & 0 & 6 & \\
\hline 0 & 0 & 6 & 6 & \\
\hline 1 & 40 & 36 & 7 & \\
\hline 2 & 80 & 72 & 0 & Crash \\
\hline 3 & 120 & 110 & N/A & \\
\hline
\end{tabular}

Table III shows the results of the second aerial test performed. The test without any attenuation produced a result of 12 targets. The score for the second run under $72 \mathrm{~dB}$ was 11 targets without any measured loss in communications. After a battery change and a first attempt, a final test was performed under $110 \mathrm{~dB}$ of measured attenuation where the operator scored 13 total targets. The results showed that the second aerial tested showed no communication issues under any number of layers of attenuation material (up to 3). This performance receives a "PASS" for the pass/fail nature of the tests performed. The second aerial robot far out-performed the first aerial robot and exceeded the available resource material to undergo further testing. This performance could have been for any number of reasons since the nature of introducing a second robot creates new factors of usability and handling for the operator but most likely was due to a better antennae and stronger signal between the OCU and the aerial robot. It did not seem that the operators ability to use either robot varied between test trials.

Table 3: Experimental Results 2

\begin{tabular}{|c|c|c|c|c|}
\hline Layers & Nom. dB & Measured dB & Performance & Notes \\
\hline-1 & 0 & 0 & 12 & \\
\hline
\end{tabular}




\begin{tabular}{|c|c|c|c|c|}
\hline 0 & 0 & 6 & Pass & Skip \\
\hline 1 & 40 & 36 & Pass & Skip \\
\hline 2 & 80 & 72 & 11 & \\
\hline 3 & 120 & 110 & 13 & $2^{\text {nd }}$ Attempt \\
\hline
\end{tabular}

\section{Future work}

Next steps would include a redesigned OCU attenuator that completely enclosed the OCU and operator to help lessen the loss of attenuation through the arms of the operator and through the holes within the initial OCU attenuator. Additionally, without first being able to isolate the test method there was no easy way to determine how much the surrounding environment was affecting the experiment. Since each layer of attenuating material provided approximately $40 \mathrm{~dB}$ of attenuation, if a robot failed at $120 \mathrm{~dB}$ but passed at $80 \mathrm{~dB}$, there was no way to tell whether or not it would have failed at any value of $\mathrm{dB}$ between these values. This leaves a large gap in information. Thinner material with more layers would provide more discretized and accurate information, but as a trade-off, it would require more testing and setup time.

\section{Conclusions}

This test will help users to consider their needs in communications when making a procurement or choosing a robot for their missions. The radio attenuation test is a necessary performance test for any response robot. A standardized radio attenuation test would offer a layer of knowledge to a first responder that has yet to be seen. Knowing whether or not a robot will perform at a certain range, or within a certain environment, before a mission begins, could be the difference between losing a robot in the field and saving a victim. Upon the initial evaluation of the test method that was performed, the general objective was successfully achieved as it showed a difference in performance between two aerial robots. The added attenuation had directly measurable performance effect on both aerials. 


\section{References}

[1] International Telecommunications Union, “ITU-R P.1238-8 Propagation data and prediction methods for the planning of indoor radiocommunication systems and radio local area networks in the frequency range $900 \mathrm{MHz}$ to $100 \mathrm{GHz}$,” International Telecommunications Union, Tech. Rep., 2015. [Online]. Available: https://www.itu.int/rec/R-REC-P.1238-8-201507-I/en

[2] T. S. Rappaport, Wireless communications: principles and practice, 2002, vol. 2. [Online]. Available: http: //www.loc.gov/catdir/toc/fy022/2002279109.html

[3] Jacoff, Adam. ASTM International. E2855-12 Standard Test Method for Evaluating Emergency Response Robot Capabilities: Radio Communication: Non-Line-of-Sight Range. West Conshohocken, PA, 2012. Web. 11 Mar 2019. <https://doi.org/10.1520/E2855-12> 